\title{
Runtime Monitoring of Timing Constraints in Distributed Real-Time Systems*
}

Department of Electrical Engineering and Computer Science, University of Michigan, Ann Arbor, MI 48109

RAGUNATHAN RAJKUMAR

rr@sei.cmu.edu

Software Engineering Institute, Carnegie-Mellon University, Pittsburgh, PA 15213

SITARAM C. V. RAJU ${ }^{* *}$

sitaram@cs.washington.edu

Department of Computer Science and Engineering, University of Washington, Seattle WA 98195

\begin{abstract}
Embedded real-time systems often operate under strict timing and dependability constraints. To ensure responsiveness, these systems must be able to provide the expected services in a timely manner even in the presence of faults. In this paper, we describe a run-time environment for monitoring of timing constraints in distributed real-time systems. In particular, we focus on the problem of detecting violations of timing assertions in an environment in which the real-time tasks run on multiple processors, and timing constraints can be either inter-processor or intra-processor constraints. Constraint violations are detected at the earliest possible time by deriving and checking intermediate constraints from the user-specified constraints. If the violations must be detected as early as possible, then the problem of minimizing the number of messages to be exchanged between the processors becomes intractable. We characterize a sub-class of timing constraints that occur commonly in distributed real-time systems and whose message requirements can be minimized. We also take into account the drift among the various processor clocks when detecting a violation of a timing assertion. Finally, we describe a prototype implementation of a distributed nun-time monitor.
\end{abstract}

\section{Introduction}

With ever-increasing reliance on digital computers in embedded real-time systems for diverse applications such as avionics, distributed process control, air-traffic control, and patient life-support monitoring, the need for dependable systems that deliver services in a timely manner has become crucial. Embedded real-time systems are in essence responsive: they often interact with the environment by "reacting to stimuli of external events and producing results, within specified timing constraints" (Kopetz and Verissimo 1993). To guarantee this responsiveness, the system must be able to provide the expected service even in the presence of faults. This paper addresses the problem of detecting timing failures in distributed real-time systems. This work is motivated by the observation that the unpredictability of the physical environment and the inability to satisfy design assumptions during transient overload can cause unexpected conditions or violations of system constraints at

\footnotetext{
* This work was done while the first two authors were at the IBM T.J. Watson Research Center.

** Supported in part by the Office of Naval Research under grant number N00014-89-J-1040 and by National Science Foundation under grant number CCR-9200858.
} 
run-time. It is highly desirable that under these error conditions, total system failure does not occur and critical system functions of the system are still performed. Hence, design assumptions and important system constraints must be monitored at run-time, and a violation must be detected and appropriate action taken in a timely fashion.

In an earlier work (Chodrow, Jahanian and Donner 1991), we have presented a general framework for formal specification and monitoring of run-time constraints in time-critical systems. We also described a single-processor implementation of a monitoring subsystem for an IBM RS/6000 workstation running the AIXv.3 operating system ${ }^{1}$. In this paper, we consider the problem of run-time monitoring in a distributed real-time system. Monitoring a timing constraint becomes more complicated in a distributed system due to the occurrences of events on multiple processors. This has several implications. First, detecting a violation as early as possible may require partial evaluation of a timing constraint as time progresses. Secondly, extra messages may have to be exchanged to propagate the occurrence of an event on a processor to others. Finally, in the absence of perfectly synchronized processor clocks or a global system clock, the meaning of a timing assertion on distributed events must be defined precisely.

Our run-time monitor for distributed real-time systems is based on the event model proposed in (Jahanian and Goyal 1990). Our prototype is an extension of the uniprocessor implementation reported in (Chodrow, Jahanian and Donner 1991). In this model, a system computation is viewed as a sequence of event occurrences. The design assumptions and system properties that must be maintained are expressed as invariant relationships between various events, which are monitored during run-time. If a violation of an invariant is detected, the system is notified so that suitable recovery options can be taken. The invariants are specified using a notation based on Real-Time Logic (RTL), and timing constraints are allowed to span processors. Our run-time monitoring facility monitors and detects violations in a distributed fashion. This distribution prevents any single monitoring process from becoming a bottleneck. In addition, monitoring of events on the processors where they occur allows violations to be detected as early as possible. Our monitor consists of a set of cooperating monitor processes (daemons), one on each processor. Application tasks on a processor inform the local monitor daemon of events as they occur. The monitor daemon on a processor checks for a violation as local events happen; it also sends the information about certain event occurrences that are needed by remote monitors to other processors. A clock synchronization algorithm ensures that event occurrence times on different processors can be meaningfully compared.

\section{I. Related Work}

Despite extensive work on monitoring and debugging facilities for parallel and distributed systems, run-time monitoring of real-time systems has received little attention with a few exceptions. Special hardware support for collecting run-time data in real-time applications has been considered in a number of recent papers (Haban and Wybranietz 1990), (Tsai, Fang and Chen 1990). These approaches introduce specialized co-processors for the collection and analysis of run-time information. The use of special-purpose hardware allows non-intrusive monitoring of a system by recording the run-time information in a large 
repository, often for post analysis. A related work (Haban and Shin 1989) studies the use of monitoring information to aid in scheduling tasks. The underutilization of a CPU due to the use of scheduling methods based on the worst-case execution times of tasks is addressed by the use of a hardware real-time monitor which measures the task execution times and delays due to resource sharing. The monitored information is fed back to the operating system for achieving an adaptive behavior. A work closer to our approach is a system for collection and analysis of distributed/parallel (real-time) programs (Kilpatrick, Schwan and Ogle 1990). The work is based on an earlier system for exploring the use of an extended E-R model for specification and access to monitoring information at run-time (Snodgrass 1988). The assumption is that the relational model is an appropriate formalism for structuring the information generated by a distributed system. A real-time monitor developed for the ARTS distributed operating system is presented in (Tokuda, Koreta and Mercer 1989). The proposed monitor requires certain support from the kernel, such as notification of the state changes of a process, including waking-up, being scheduled. In particular, the ARTS kernel records certain events that are seen by the operating system as the state of a process changes, e.g., waking-up from a blocked state, being scheduled. These events are sent periodically by the local host to a remote host for displaying the execution history. The invasiveness of the monitoring facility is included in the schedulability analysis. Monitoring and detecting violations of certain predefined timing constraints have been proposed in real-time languages, such as FLEX (Kenny and Lin 1991).

Detecting a violation of a timing assertion in a distributed system is also related to the problem of detecting stable (global) properties of a system. Many snapshot algorithms for establishing a global consistent system state have been proposed in the past (e.g. (Chandy and Lamport 1985), (Koo and Toueg 1987)). A more recent work proposed a method for detecting locally stable properties by constructing substates of a system (Marzullo and Sabel 1992). The goal of the snapshot algorithms is to preserve causality when constructing a global system state. In our case, if a history of event occurrences is maintained, then detecting a violation of a timing assertion can be viewed as detecting a stable property. Of course, a primary motivation for our work is to detect a violation as early as possible. Furthermore, causality between event occurrences is captured by a static constraint graph in our model. Recent work on evaluating nonstable global predicates for distributed computations also relate to our work, but to a lesser extent (Garg and Waldecker 1992), (Marzullo and Neiger 1991). Reference (Marzullo and Neiger 1991) looks at several techniques for limiting the exponential number of states that must be considered to evaluate a property over computations. Reference (Garg and Waldecker 1992) considers an alternative approach by restricting the global predicate to one that can be efficiently detected, such as the conjunction and disjunction of local predicates. A good article on monitoring distributed computations for asynchronous systems appears in (Babaoglu and Marzullo 1993).

The rest of this paper is organized as follows. Section 2 describes an aircraft tracking system and discusses issues in detecting a violation of a timing assertion. Section 3 presents our event-based computation model, and discusses specification of timing assertions. Section 4 presents a solution to the problem of minimizing extra messages to propagate event occurrences to other processors, and discusses the effect of synchronized clocks on detecting a violation. Section 5 describes a prototype implementation of a monitor for a network 
of RS/6000s running AIXv.3 operating system. Finally, Section 6 presents our concluding remarks.

\section{Motivation and Research Issues}

In a distributed real-time system, there can be timing constraints imposed on events across multiple processors. These interprocessor timing constraints are among the hardest to enforce as well as to verify. This section motivates the problem by describing an aircraft tracking system with end-to-end requirements.

\subsection{Aircraft Tracking System}

The structure of an aircraft tracking system is shown in Figure 1. A radar signal is received by a radar controller, which is a special-purpose processor. The signal is fed into a generalpurpose processor that does calibration and tracking. Next, the signal is sent to a host interface that does some preprocessing and sends the signal to a console processor ${ }^{2}$, which performs some filtering and number-crunching in parallel. Finally, the signal is sent to a special-purpose display processor that displays the appropriate tracking information on the monitor. There is a 2 seconds end-to-end deadline from the time an event is received by the radar controller to the time it is displayed by the display processor. In addition, there is an intermediate 0.5 second deadline on the display commands step on the display processor from the preprocessing step on the host interface processor.

\subsection{Issues in Monitoring of Distributed Constraints}

A monitoring facility can prove very useful in checking interprocessor timing constraints such as those of the aircraft tracking system. A recovery task can be invoked if a violation of timing constraints is detected. Several issues need to be addressed by a distributed monitor that checks interprocessor timing constraints.

- Time of detection of violations: Detecting violations as early as possible is a desirable property because it can allow the system to take corrective action before the violation actually happens.

- Number of messages: Since events happen on different processors and timing constraints can span processors, some form of interprocessor communication is needed to propagate this information. In a distributed environment, event occurrences must be communicated using messages. Minimizing the number of extra messages is crucial for reducing overhead.

- Clocks and Timer Granularity: When an event occurs there must be a way of recording the occurrence time of the event. The granularity of timestamping determines the minimum observable spacing between two consecutive events on a processor. Timestamping is typically done by reading the clock on the local processor. A distributed 

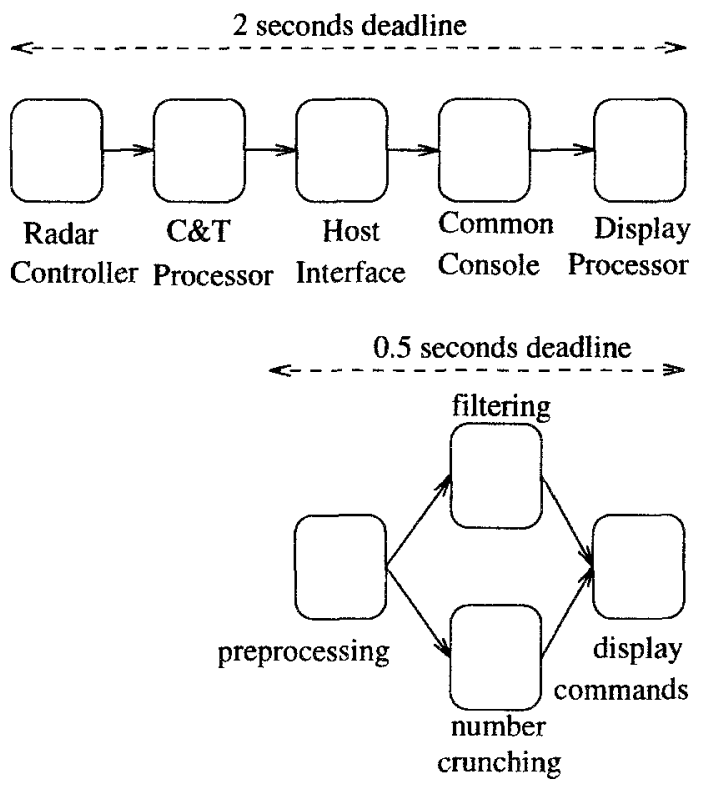

Figure 1. An Aircraft Tracking System

system must also deal with the fact that the clocks on different processors are not perfectly synchronized. The processor clocks, however, can be kept synchronized within a known maximum bound on the deviation between them. Clock synchronization allows controlled comparison of timestamps from different clocks. In particular, one must take into consideration the deviation between clocks when evaluating a timing assertion at run-time.

- Resource management: Another fundamental aspect of a distributed monitor involves the need to quantify the timing intrusiveness of the monitoring activities on the timing behavior of the real-time application. A discussion of our approach to scheduling the monitoring activities is beyond the scope of this paper and interested readers are referred to (Jahanian and Rajkumar 1991). This approach allows the run-time monitoring processes to be scheduled as time-constrained activities and therefore can be part of the schedulability test for the system.

\section{Timing Constraints in Real-Time Systems}

We express the timing constraints in a notation (Chodrow, Jahanian and Donner 1991) based on Real-Time Logic (RTL). A detailed description of the computation model and the specification language is beyond the scope of this paper. In this section, we present an informal overview of the computational model and then discuss the representation of a 
timing assertion as a directed constraint graph. Finally, we introduce the conditions under which a timing assertion can be violated.

\subsection{Specification of Timing Assertions}

An application consists of a set of cooperating tasks, perhaps running on multiple processors. The monitoring subsystem views a computation of the system as a partially-ordered sequence of event occurrences. Informally, events represent things that happen in a system. For example, an event may denote the start/completion of a program segment, reading a new sensor value into a program variable or receiving a message from another task. An event occurrence defines a point in time at which a particular instance of the event happens in a computation. Thus, a safety property or a timing constraint can be expressed as an assertion about the relationship between event occurrences in a computation. For example, suppose event $E$ denotes the invocation of a task $T$ and another event $E^{\prime}$ denotes the completion of the task, and task $\mathrm{T}$ is executed periodically. Each invocation of the task corresponds to an occurrence of the event $E$. Similarly, when a particular invocation of task T completes, it corresponds to an occurrence of event $E^{\prime}$. A timing constraint, such as a deadline, specifies the relationship between the occurrences of event $E$ and $E^{\prime}$. A deadline of $100 \mathrm{~ms}$ on the execution of task $T$ requires that the corresponding occurrences of $E$ and $E^{\prime}$ should be within $100 \mathrm{~ms}$.

To detect timing constraints, it may be necessary to record multiple occurrences of an event. For example, to check a constraint on the interval between two successive instances of an event, the past two instances of the event must be stored. The occurrences of an event are stored in a circular queue called its event history, which maintains the last $n$ occurrence times of the event. The value of $n$ depends on the assertion.

In our model, a timing requirements on a system is viewed as an assertion on the events that can occur in the system. We need to provide a notation for specifying complex constraints that are to be monitored at run-time. The notation must be expressive enough to capture a variety of complex constraints that may be imposed on a system. For example, a timing constraint may specify an end-to-end deadline on a set of actions that must be executed upon receiving a new sensor value or a safety assertion may require a specific precedence must hold among the actions while the system is in a certain mode. As described in (Jahanian and Goyal 1990), we use a notation based on RTL for specifying timing assertions. We provide two functions for accessing the event histories: the occurrence function $@(e, i)$, which returns the time of the $i$ th occurrence of event $e$, and @val $(v, i)$, which returns the value of the $i$ th assignment to a variable $v$. A positive occurrence index is absolute with respect to the beginning of the computation sequence. For example, $@(e, 5)$ refers to the 5 th occurrence of event $e$. When the index $i$ is negative, it refers to the $i$ th most recent occurrence of the event in a computation. For example, $@(e,-1)$ denotes the time of the most recent occurrence of $e$. An occurrence index of 0 is undefined. We present two examples to illustrate how the notation can be used. 
Example: Consider two events $e_{1}$ and $e_{2}$ which must always occur in pairs and within 5 time-units of each other. The following formula specifies such a constraint.

$$
\forall i @\left(e_{1}, i\right) \leq @\left(e_{2}, i\right)+5 \vee @\left(e_{2}, i\right) \leq @\left(e_{1}, i\right)+5
$$

Example: The value of the occurrence indices in a timing constraint need not be a variable or a positive integer. It can be relative to the current index in a computation. Consider an event whose successive occurrences must be separated by at least 5 time-units. The following RTL formula specifies such a constraint.

$$
@(\text { response, }-2) \leq @(\text { response, },-1)-5
$$

If this constraint is checked whenever a response event occurs, then it is equivalent to

$$
\forall i>1 @(\text { response, } i-1) \leq @(\text { response, } i)-5
$$

If a violation occurs, the system will be notified by the monitoring facility. The checking of a timing constraint may require multiple instances of an event occurrence to be stored. Each of these occurrences is referred to by an occurrence index.

\subsection{Graph Representation of Timing Constraints}

If a timing assertion is in a disjunctive normal form as in Equation 1, each conjunct can be represented as a directed, weighted graph, called a constraint graph. Each constraint graph represents a conjunction of predicates, and each edge in the graph is a predicate of the form:

$$
@(e, i) \leq @(f, j) \pm C
$$

such that $i, j$ are integer variables/constants ${ }^{3}$ and $C$ is an integer constant.

Intuitively, a predicate in a conjunct represents either a delay or a deadline constraint on a pair of events. The vertices of the constraint graph correspond to unique occurrence functions; the weighted edges denote the constraints between event pairs. For example, the following delay constraint:

$$
@(\text { response },-2) \leq @(\text { response, }-1)-5
$$

is represented by an edge with weight -5 as shown in Figure 2. A predicate of the form @(response, 1$) \leq C$ where $C$ is an absolute time value is translated to an edge $\mathbf{0} \stackrel{C}{\longrightarrow} @($ response, 1$)$ where $\mathbf{0}$ is a special "zero vertex" designed to take care of constants. Similarly, a predicate of the form $C \leq @($ response, 1$)$ is represented by an edge $@($ response, 1$) \stackrel{-C}{\longrightarrow} \mathbf{0}$.

As an example of a deadline constraint, consider the following assertion:

$$
\forall i @(a c k, i) \leq @(\text { send }, i)+12
$$




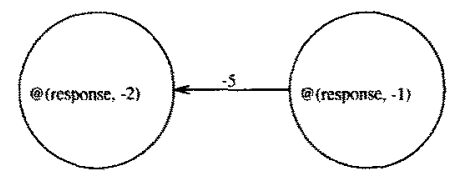

Figure 2. Delay constraint

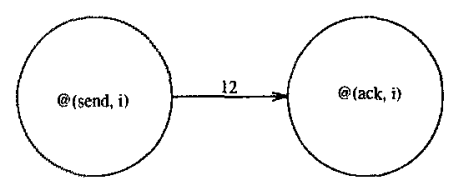

Figure 3. Deadline constraint

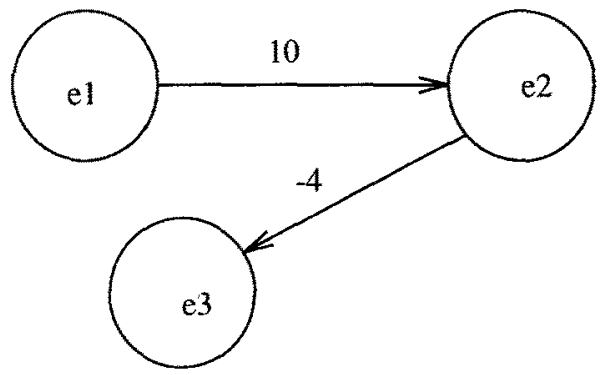

Figure 4. Intermediate deadline from $e l$ to $e 3$

This constraint specifies that an ack event must occur within 12 time-units of its corresponding send event, and is represented by an edge with positive weight 12 as shown in Figure 3.

A path between two vertices $u$ and $v$ in the graph is a sequence of edges from $u$ to $v$. The length of a path is the sum of the weights of all edges along the path. In the rest of the paper, without loss of generality, we do not associate any occurrence indices to events. This is possible because a specific constraint graph is instantiated for a set of event occurrence indices before checking for a violation.

\subsection{Implicit Constraints}

In addition to the explicit delay or deadline edges in a constraint graph, we can derive certain implicit constraints often as an intermediate deadline or delay. In fact, it is possible that an implicit constraint is violated before an explicit deadline or delay becomes unsatisfiable at run-time.

For example, consider the simple constraint graph in Figure 4. It consists of two explicit timing constraints: a deadline edge and a delay edge. Events $e 1, e 2$ and $e 3$ occur on 
processors 1, 2 and 3 , respectively. There is an explicit deadline from $e 1$ to $e 2$. In addition, since there is a path from $e l$ to $e 3$ of length 6 , there is an implicit, intermediate deadline of 6 from $e 1$ to $e 3$. If the intermediate deadline is not met, then either the explicit deadline or the delay constraint from $e 3$ to $e 2$ will eventually be violated. If the violation of the implicit constraint between $e I$ to $e 3$ is detected, the system can be notified before any of the two user-specified constraints are violated. As a result, corrective action can potentially be taken even before the application-level constraint is violated.

\subsection{Checking Constraint Graphs}

The constraint graphs must be checked for potential violations at certain discrete points in time. We establish these discrete points of time in this section.

When an event occurs that may affect the satisfiability of a timing assertion, a satisfiability checker is invoked to check for violations. The checker instantiates the vertices of the graph from event histories. For example, the vertex @(response, -1 ) will be replaced by the occurrence time of the most recent response event. The vertex @(send, $i$ ) will be replaced by the occurrence time of the current activation of send event. Vertices that have been instantiated are merged with the $\mathbf{0}$ vertex as follows: Every edge with weight $w$ incoming to a vertex, to which a time value $t$ has been assigned, is replaced with an edge with weight $w-t$ incident on the $\mathbf{0}$ vertex. Conversely, every edge with weight $w$ outgoing from a vertex, to which time $t$ has been assigned, is replaced with an edge of weight $w+t$ outgoing from the 0 vertex. Vertices that have not yet occurred are not instantiated. Instead, an edge from the uninstantiated vertex to the 0 vertex, of weight equal to -current_time is added. This is an assertion that the event has not happened since system startup. The actual algorithm for checking violations will be discussed in Section 4.2 .

Event occurrences in real-time systems can happen in different orders. For example, two occurrences each of events $A$ and $B$ can occur as (among other possibilities)

$$
A_{1} B_{1} A_{2} B_{2}
$$

or as,

$$
A_{1} A_{2} B_{1} B_{2}
$$

If there is a deadline constraint from the $i^{t h}$ occurrence of $A$ to the $i^{\text {th }}$ occurrence of $B$ then the timing constraint is from $A_{1}$ to $B_{1}$ and from $A_{2}$ to $B_{2}$. Hence when the constraint graph is instantiated, the same occurrence index must be used for all vertices in the constraint graph.

We state a lemma that establishes the conditions under which a timing assertion (constraint graph) may be violated.

LEMMA 1 In a constraint graph, the earliest time a constraint can be violated is as follows:

1. A delay constraint will be violated, if for a path of negative length $-T(T \geq 0)$ from vertex $e_{n}$ to vertex 0 , the event corresponding to vertex $e_{n}$ happens before time $T$. 
2. A deadline constraint will be violated if the minimum length $T(T \geq 0)$ of all shortest paths from vertex 0 to all other vertices is to a vertex $e_{m}$ and the event corresponding to vertex $e_{m}$ does not happen at or before $T$.

Proof: The proof of Lemma 1 appears in the appendix.

Lemma 1 states that delay violations need only be tested whenever an event occurs, and deadline violations need not be tested before some timeout value. Hence, a constraint graph must be checked for violations after the occurrence of any event in the graph. The event occurrence is instantiated in the graph with its occurrence time and the graph is checked for violations. If the graph is not violated, the length of the minimum of the shortest paths from vertex 0 to all uninstantiated vertices is computed. If this length $P$ is not infinity then a timer that expires at time $P$ is set. The graph is again checked for violations when the timer expires or when an event happens, whichever is earlier.

\section{A Monitor for Distributed Real-Time Systems}

In this section, we focus on our approach to deal with the issues that arise in monitoring distributed real-time systems such as the aircraft tracking system of Figure 1.

We assume that interprocessor communication is reliable. This assumption is valid whenever a reliable communication mechanism based on acknowledgments is used. We also assume that there is no migration of application tasks among processors. This assumption can be relaxed if as part of a mode change, the constraints to be monitored and the new communication patterns are also re-established. In this section, we also assume that if there is a delay constraint between a pair of events on distinct processors, the communication latency between the two processors is less than the delay constraint.

In the preceeding section, we established the conditions under which a timing assertion may be violated. We now focus on two other key problems: minimization of messages given that violations must be detected as early as possible, and the effect of clock synchronization in evaluating a constraint at run-time.

\subsection{Minimization of Messages}

Messages must be passed across processor boundaries to check interprocessor timing constraints. In this subsection, we address the issue of minimizing these message-passing requirements. We assume that each monitor process knows every constraint graph that contains one or more events happening on its local processor. Whenever a local event occurs, a monitor process must decide whether the event must be communicated to other monitors. We also assume in this subsection that there is a single clock in the system. This assumption will be relaxed in the next subsection.

We shall use the following terminology. There is a correspondence between an event $e_{n}$, the processor $n$ on which the event happens and the monitor on processor $n$. Hence, we 
will use the phrase $e_{i}$ 's monitor to mean the monitor local to the processor on which $e_{i}$ occurs.

Given a constraint graph $G$ and a vertex $e_{i}$, the list of monitors to whom the occurrence of $e_{i}$ must be communicated can be determined as follows. Run the shortest-path algorithm on the graph $G$ such that the shortest path from any vertex to every other vertex is obtained. We refer to this resulting graph as the shortest path graph. This transformation of the constraint graph adds edges that represent implicit constraints. Messages with event occurrence times may also need to be sent over these additional edges. The shortest path graph captures both explicit and implicit constraints. Since implicit constraints are derived from explicit constraints, the violation of an implicit constraint implies the (potentially future) violation of an explicit constraint. To detect violations as early as possible, implicit constraints need to be considered.

Whenever an event $e_{i}$ occurs, its occurrence needs to be communicated (directly or indirectly) to the monitor of any vertex $e_{j}$, if in the shortest path graph, there exists a path with positive weight from $e_{i}$ to $e_{j}$ or a path with negative weight from $e_{j}$ to $e_{i}$. This procedure is illustrated in Figure 5(a), which shows a constraint graph $G$. The shortest-path graph derived for one vertex $e_{1}$ is shown in Figure 5(b). From this graph, whenever event $e_{1}$ occurs, it must be communicated (directly or indirectly) to the monitors of events $e_{2}$ through $e_{6}$.

In practice, instead of running the shortest-path algorithm w.r.t. one node, the algorithm would be run on the entire graph such that the shortest path from any node to every other node is obtained.

1. Delay violations. If there is a vertex $e_{j}$ such that there exists a path from $e_{j}$ to $e_{i}$ of negative length, then there is a precedence constraint between $e_{i}$ and $e_{j}$. The occurrence time of $e_{i}$ must be sent to $e_{j}$ 's monitor, so that when $e_{j}$ happens the monitor can check if the delay constraint has been violated. Hence the occurrence time of $e_{i}$ must be sent to all such monitors. Thus, in Figure $4, e_{3}$ 's occurrence time needs to be sent to $e_{2}$ 's monitor.

2. Deadline violations. If there is a vertex $e_{j}$ such that there exists an edge with positive weight from $e_{i}$ to $e_{j}$, then the occurrence time of $e_{i}$ must be sent to $e_{j}$ 's monitor, so that the monitor can check if $e_{j}$ happens within the deadline. There may be events $e_{k}$ that precede $e_{j}$, but not $e_{i}$. Such events will have earlier deadlines and represent intermediate points at which eventual violations of delays/deadlines can be detected. Hence messages must also be sent to all such $e_{k}$ 's monitors. For example, in Figure $4, e_{1}$ 's occurrence time needs to be sent to $e_{2}$ 's monitor and to $e_{3}$ 's monitor. The requirement of earliest violation detection can be relaxed, to reduce the number of messages. In this case, the occurrence time needs to be sent only to all $e_{j}$ 's monitors such that there exists an edge with positive weight from $e_{i}$ to $e_{j}$.

Using the shortest path graph to determine the recipient monitors of an event occurrence can be very pessimistic. It is often possible that some of the messages can be eliminated as they are either redundant or two (or more) messages can be combined into a single message. For example in Figure 5(b), $e_{1}$ 's occurrence needs to be communicated to $e_{4}$ 's monitor and to $e_{5}$ 's monitor. Also, $e_{4}$ 's occurrence time needs to be communicated to $e_{5}$ 's monitor. 


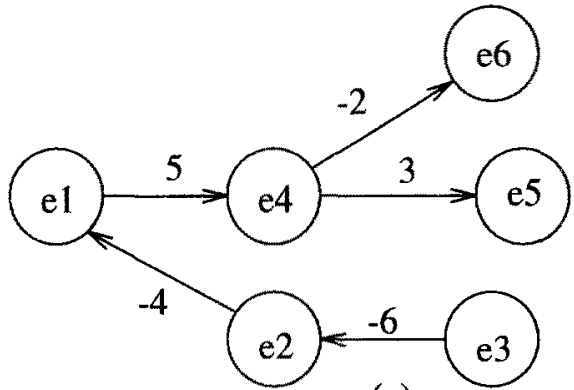

(a)

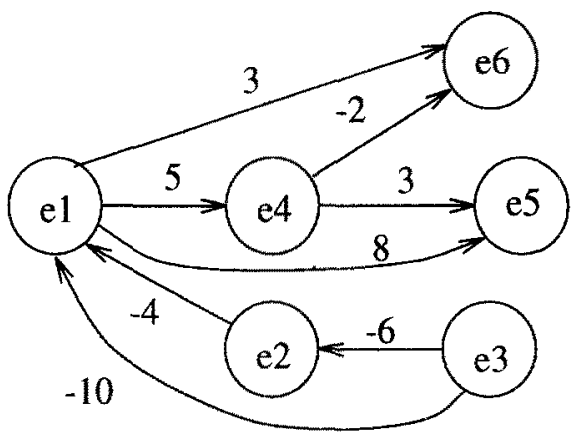

(b)

Figure 5. Determining the recipients of event $e_{1}$ (a) The application-level constraint graph. (b) The shortest path constraint graph for $e_{1}$.

However, the weight of the edge from vertex $e_{1}$ to vertex $e_{5}$ is the same as the length of the path $e_{1} e_{4} e_{5}$. As a result, the message from $e_{1}$ 's monitor to $e_{5}$ 's monitor, containing the occurrence time of $e_{1}$, can be eliminated.

Again in Figure 5(b), $e_{1}$ 's occurrence time needs to be sent to the monitors of $e_{2}$ and $e_{3}$. In addition, $e_{2}$ 's occurrence time needs to be sent to $e_{3}$ 's monitor. There is also an ordering of events, $e_{1}$ (first), $e_{2}$ (second) and $e_{3}$ (last) such that if these events happen in any other order, a constraint would be violated. As a result, the message from $e_{2}$ 's monitor to $e_{3}$ 's monitor can also carry the occurrence time of $e_{1}$. Thus, the message from $e_{1}$ 's monitor to $e_{3}$ 's monitor can be eliminated.

Naturally, it is desirable that the maximum number of messages are either removed or combined. However, the problem of minimizing the number of messages for arbitrary constraint graphs is intractable. We show next that removing the maximum number of redundant messages is NP-complete for constraint graphs whose edges have positive weights only. The formal statement of the problem called irredundant deadline graph (IDG) problem is as follows:

Instance: Given a constraint graph $G$ with positive weights for all edges, and a positive integer $K \leq$ number of edges in $G$. 
Question: Is there a subset $G^{\prime} \subseteq G$ where the number of edges in $G^{\prime}$ is $\leq K$ such that, for every ordered pair of vertices, $u, v \in G$, the shortest path from $u$ to $v$ in graph $G^{\prime}$ is of length $d$, if and only if the shortest path from $u$ to $v$ in $G$ is also of length $d$ ?

\section{THEOREM 1 Irredundant deadline graph (IDG) is $N P$-complete.}

Proof: By transformation from the minimum equivalent digraph problem (MED) (Garey and Johnson 1979). The formal statement of the MED problem is as follows:

Instance: Directed graph $G=(V, A)$, positive integer $K \leq|A|$.

Question: Is there a subset $A^{\prime} \subseteq A$ with $\left|A^{\prime}\right| \leq K$ such that, for every ordered pair of vertices $u, v \in V$, the graph $G^{\prime}=\left(V, A^{\prime}\right)$ contains a directed path from $u$ to $v$ if and only if $G$ does?

A nondeterministic algorithm can guess a set of $\left|K^{\prime}\right|$ edges, compute the all pairs shortest paths for $G$ and $G^{\prime}$ and check them for equality. Hence IDG is in NP.

We can transform an arbitrary instance of MED, $M=(V, A)$, into an instance of IDG $G^{\prime}=\left(V, A^{\prime}\right)$, by assigning a weight of 0 to every edge of $M$. This can be done in polynomial time. Clearly, the irredundant deadline graph for $G^{\prime}$ will have a path between any ordered pair of vertices $u, v$ if and only if $M$ has a path between $u, v$. Hence the answer to IDG is yes if and only if the answer to the corresponding MED is yes. Hence IDG is NP-complete.

Given that the problem of minimizing the number of messages is intractable, we next consider sub-classes of constraint graphs that are likely to occur in real-time systems, and whose message requirements can be easily minimized.

Definition: An event $e_{i}$ is said to precede event $e_{j}$ in a constraint graph, if there exists a path from vertex $e_{j}$ to vertex $e_{i}$ that consists of delay edges (i.e., edges with negative weights) only.

Given this definition, we use the terms "delay edges" and "precedence edges" interchangeably.

\subsubsection{Precedence-Preserving Graphs}

The delay edges impose precedence or partial ordering on the set of event occurrences in a constraint graph. Intuitively, the delay edges may represent the computation time of a task or causality between a pair of events. For example, if there is a delay from event $e_{1}$ to $e_{2}$ (a negative edge from $e_{2}$ to $e_{1}$ ), event $e_{1}$ must occur before (precede) $e_{2}$. Otherwise, the delay constraint is violated. In real-time systems, it is common to have an event that triggers a task execution, which in turn generates other events. For example, in the aircraft tracking system described in Section 2, the computation in each module triggers the computation in the next module on completion. An incoming track signal is processed by different modules in pipelined fashion. As a result, every data item that crosses module/processor boundaries has delay or precedence constraints, and deadline constraints tend to be end-toend deadlines. 


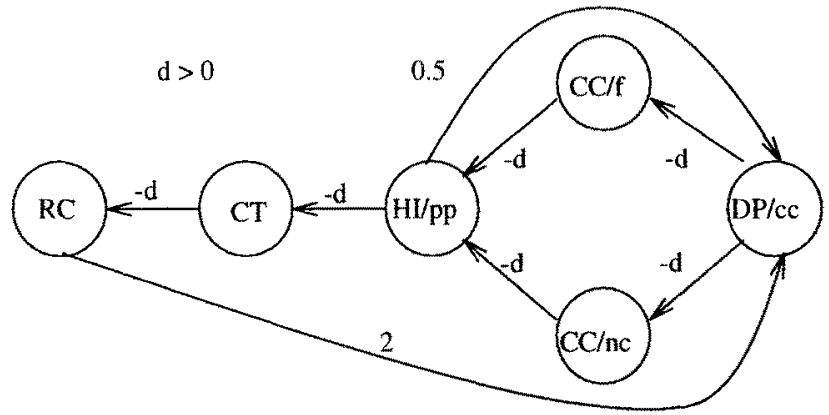

Figure 6. The Constraint Graph for the Tracking System of Figure 1

In general, precedence relations are natural in real-time systems where data streams are processed in pipeline stages. Consider a distributed audio/video system that processes its data in pipelined stages from one node to another node via gateways and communication networks. More specifically, the audio/video signal is digitized and compressed at a sender node and transmitted to a receiver node where it is uncompressed and displayed. The audio/video data must be received/displayed at the receiver node at a precise rate. Hence, there are precedence constraints between the various stages. If the data corresponds to live interaction such as video conferencing, latency requirements will force an end-to-end deadline as well.

Precedence relations among events also exhibit desirable properties from the communication requirements viewpoint. For example, if event $e_{1}$ precedes event $e_{2}$, then $e_{2}$ 's monitor must receive the occurrence time of $e_{1}$ before $e_{2}$ occurs. Otherwise a violation has taken place. Hence, $e_{2}$ 's monitor always has the potential to send the occurrence time of $e_{1}$ and $e_{2}$ in a single message to a third monitor. These combinations can save messages. We present a sub-class of constraint graphs called precedence-preserving graphs where sending messages only along the delay edges is sufficient to detect all violations at the earliest possible time.

Definition: A precedence-preserving graph is a constraint graph that satisfies the following condition: If there is a shortest path from vertex $e_{i}$ to vertex $e_{j}$ of positive length, then the source vertices of deadline edges on the path precede $e_{j}$.

Recall the aircraft tracking system described in Section 2.1. The corresponding constraint graph, shown in Figure 6, is a precedence-preserving graph. The 2 seconds deadline edge is between the $R C$ and $D P / c c$ vertices, where the former precedes the latter. Also, the 0.5 seconds deadline is between the $H I / p p$ and $D P / c c$ vertices, which also have a precedence ordering. As a result, in this graph, messages need be sent only along the delay edges. For example, the node $R C$ will send its event only to node $C T$. If the shortest path graph were used, there exists a positive path from $R C$ to every other node so that $R C$ would have to send 5 messages. The precedence-preserving graph thus results in substantial savings of messages without compromising the time at which a violation will be detected. We now prove this property of precedence-preserving graphs. The theorem is based on the 


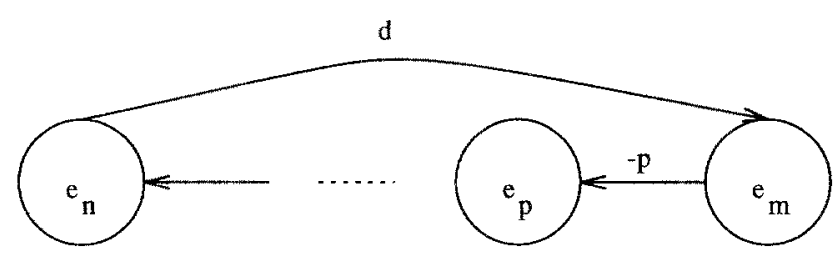

Figure 7. Case where $e_{n}$ precedes $e_{m}$

assumption that the message communication time between any two processors is less than the delay constraints between events on the two processors. We also assume that a message carries the time of occurrence of the local event and its predecessor events.

THEOREM 2 In a precedence-preserving graph, if messages are being sent only along the precedence edges, then all violations will be detected at the earliest possible time.

Proof: The proof consists of showing that messages along precedence edges carry the required information of event occurrences to a vertex before delay or deadline violations can occur.

Delay violation: Consider a vertex $e_{n}$ in a precedence-preserving graph. A delay constraint to another vertex $e_{d}$ can be of 2 types:

1. there is a negative edge from $e_{d}$ to $e_{n}$.

2. there is a path containing negative edges only from $e_{d}$ to $e_{n}$.

In the first case the occurrence time of $e_{n}$ is sent to $e_{d}$. Delay violations can be detected according to Lemma 1 . In the second case there are 2 possibilities:

1. The immediate predecessor of $e_{d}$ in the constraint graph has already occurred. Hence $e_{d}$ will have information about the occurrence time of the immediate predecessor and its predecessors. Hence, constraint violations, if any, can be detected by Lemma 1.

2. The immediate predecessor of $e_{d}$, say $e_{i}$, has not happened. This implies that the delay constraint to $e_{i}$ has been violated. Hence the occurrence time of $e_{i}$ and its predecessors is irrelevant.

Deadline violation:

LEMMA 2 If there is a deadline from vertex $e_{n}$ to vertex $e_{m}$, then either $e_{n}$ precedes $e_{m}$ or there exists a vertex $e_{k}$ that precedes both $e_{n}$ and $e_{m}$.

Proof of Lemma 2: Consider the path from $e_{n}$ to $e_{m}$. Let the path be $e_{n}, e_{i}, e_{i+1}, e_{i+2}$ $\ldots e_{i+p}, e_{m}$. If the edge from $e_{n}$ to $e_{i}$ is positive, then from the definition of precedencepreserving graphs, $e_{n}$ must precede $e_{m}$. If the edge from $e_{n}$ to $e_{i}$ is negative, then consider the first positive edge in the path (there must be such an edge as sum of weights on the 


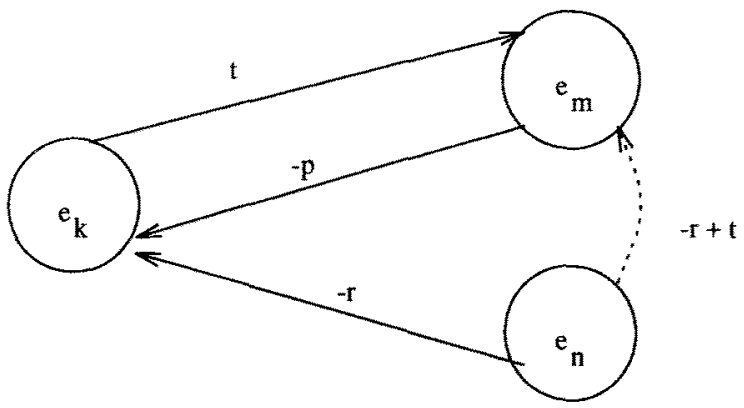

Figure 8 . Case where $e_{k}$ precedes $e_{n}$ and $e_{m}$

path is positive). Say the positive edge is from $e_{i+a}$ to $e_{i+a+1}$. Now, from the definition of precedence oriented graphs, $e_{i+a}$ precedes $e_{m}$. Also since there is a sequence of negative edges from $e_{n}$ to $e_{i+a}, e_{i+a}$ precedes $e_{n}$. Thus, $e_{i+a}$ is the vertex $e_{k}$.

Consider the constraint graph of Figure 7 where $e_{n}$ precedes $e_{m}$. Let $e_{p}$ be the immediate predecessor of $e_{m}$. Let $-p(p>0)$ be the weight of edge from $e_{m}$ to $e_{p}$. Hence there is a deadline $d-p$ on $e_{p}$. Since,

$$
d-p<d
$$

the deadline on $e_{p}$ is shorter than the deadline on $e_{m}$. Hence the occurrence time of $e_{n}$ needs to be communicated to $e_{p}$ first. When $e_{p}$ occurs, the occurrence time of $e_{p}$ and its predecessors events will be communicated to $e_{n}$. By extending this argument to $e_{p}$ 's immediate predecessor and so on, it can be seen that messages need to be sent along precedence edges only.

Now consider the constraint graph of Figure 8 where a vertex $e_{k}$ precedes $e_{n}$ and $e_{m}$. Consider the deadline from $e_{n}$ to $e_{m}$.

$$
\text { deadline }=@\left(\mathrm{e}_{\mathrm{n}}\right)-\mathrm{r}+\mathrm{t}
$$

The deadline from $e_{k}$ to $e_{m}$ is

$$
@\left(\mathrm{e}_{\mathrm{k}}\right)+\mathrm{t}
$$

Assume,

$$
@\left(e_{n}\right)-r+t<@\left(e_{k}\right)+t
$$

then,

$$
@\left(e_{n}\right)<@\left(e_{k}\right)+r
$$


But this means that the delay constraint from $e_{k}$ to $e_{n}$ is violated and it will be detected by $e_{n}$. In the absence of violation, the deadline on $e_{m}$ is not shortened. Hence, messages along the precedence edges will suffice.

If a constraint graph is not a precedence-preserving graph, its message requirements cannot be (easily) minimized but they can still be reduced. An edge $E$ from vertex $e_{i}$ to vertex $e_{j}$ can be deleted from a shortest-path constraint graph $G_{s}$, if the weight on the edge is greater than or equal to the shortest path from $e_{i}$ to $e_{j}$ in the graph $G_{s}-E$. If an edge can be removed, then the corresponding message along the edge need not be transmitted. As a result, given an arbitrary constraint graph $G$, the messages that need to be communicated can be determined as follows:

1. Run the shortest-path algorithm on $G$ to obtain its shortest-path constraint graph $G_{s}$. Let $G^{\prime}=G_{s}$.

2. Pick an edge $E$ in $G^{\prime}$ from vertex $e_{i}$ to vertex $e_{j}$ such that the following condition is satisfied. If the edge has a positive (negative) weight, the out-degree (in-degree) of $e_{i}$ and the in-degree (out-degree) of $e_{j}$ must be greater than 1 . If there is no such edge, the procedure ends.

3. Find the length of the shortest path $p$ from vertex $e_{i}$ to vertex $e_{j}$ in $G^{\prime}-E$.

4. If $p \leq$ weight on $E$, delete $E$ from $G^{\prime}$.

5. Go to Step 2 .

The final graph $G^{\prime}$ determines what messages must be transmitted when an event corresponding to a vertex occurs. Messages must be sent from a vertex $e_{i}$ to a vertex $e_{j}$ if there is a positive edge from $e_{i}$ to $e_{j}$ or a negative edge from $e_{j}$ to $e_{i}$.

\subsection{Effect of Approximately Synchronized Clocks}

The occurrence time of an event corresponds to the local time at its time of occurrence on the processor where the event occurs. In a distributed system, the clocks on different processors are not identical and deviate from one another. As a result, the checking of constraint graphs must take these clock deviations into account.

If the deviation between various clocks is not bounded by a known value, it is difficult (perhaps impossible) to enforce in a meaningful way a timing constraint whose events span multiple processors. We therefore use a clock synchronization algorithm to bound the deviations among the various processor clocks (Arvind 1989), (Cristian 1989), (Lundelius and Lynch 1984). As discussed in a subsequent section, we implemented approximately synchronized clocks based on the probabilistic algorithm described in (Cristian 1989). A clock process on each processor synchronizes with the clock process on a master processor (master clock) by exchanging messages.

Let $\epsilon$ be the maximum deviation among the clocks. We now state a theorem that gives the necessary and sufficient condition for violations in a constraint graph in which a constraint 
spans events on more than one processor. The intuition behind the theorem is as follows: If there is a constraint of length $P$ between events on two different processors, the constraint length should be changed to $P-\epsilon$ within the system. For example, if event $e_{2}$ must happen within 10 time-units after event $e_{1}$, the bounded clock deviation requires that $e_{2}$ actually happen within $10-\epsilon$ after $e_{1}$. Otherwise, a violation may have occurred.

THEOREM 3 Given a cycle spanning more than one processor in a constraint graph, there exists a set of clock deviations for which a constraint is violated, if and only if the length of the cycle is less than $\epsilon$.

Proof: Since there is a cycle spanning more than one processor, there must exist two adjacent vertices $e_{i}$ and $e_{j}$ in the cycle that are on two different processors.

If part. Let there be a cycle of length $<\epsilon$. We consider delay and deadline constraints between $e_{i}$ and $e_{j}$, and show that in either case the constraint is violated. The occurrence time of any event is the time of its occurrence according to its local clock. Also, the local clock of an event refers to the clock of the processor on which the event happens.

1. Delay constraint of $P(0<P)$ between $e_{i}$ and $e_{j}$, i.e. $e_{j}$ must occur any time at or after $P$ time-units after the occurrence of $e_{i}$. This means that

$$
@\left(e_{i}\right)+P \leq @\left(e_{j}\right)
$$

Let the local clock of $e_{j}$ be $\epsilon$ ahead of the local clock of $e_{i}$. A violation takes place if $e_{j}$ occurs on $e_{j}$ 's processor any time before $P+\epsilon$ after $e_{i}$ 's occurrence. Hence, the constraint between the events must be adjusted as

$$
@\left(e_{i}\right)+(P+\epsilon) \leq @\left(e_{j}\right)
$$

Since there is a cycle, there has to be a path from $e_{i}$ to $e_{j}$. Let the length of this path be $Q$. That is, $e_{j}$ must occur any time at or before $Q$ time-units after the occurrence of $e_{i}$. This means that

$$
@\left(e_{j}\right) \leq Q+@\left(e_{i}\right)
$$

For Equations (2) and (3) to be satisfied, we must have

$$
Q-P \geq \epsilon
$$

This contradicts our assumption that the cycle length is less than $\epsilon$, i.e. $Q-P<\epsilon$.

2. Deadline constraint of $P(0<P)$ between $e_{i}$ and $e_{j}$, i.e. $e_{j}$ must occur any time at or before $P$ time-units after the occurrence of $e_{i}$. This means that

$$
@\left(e_{j}\right) \leq @\left(e_{i}\right)+P
$$


Let the local clock of $e_{j}$ be $\epsilon$ behind the local clock of $e_{i}$. A violation takes place if $e_{j}$ occurs any time after $P-\epsilon$ after $e_{i}$. Hence, the constraint between the two events must be adjusted as

$$
@\left(e_{j}\right) \leq @\left(e_{i}\right)+(P-\epsilon)
$$

Since there is a cycle, there has to be a path from $e_{j}$ to $e_{i}$ of length $-Q$. This means that,

$$
@\left(e_{i}\right)+Q \leq @\left(e_{j}\right)
$$

For Equations (6) and (7) to be satisfied, we must have

$$
P-Q \geq €
$$

This contradicts our assumption that the cycle length is less than $\epsilon$, i.e. $P-Q<\epsilon$.

Only if part. Let a constraint between vertices $e_{i}$ and $e_{j}$ be violated. The constraint can either be a delay or a deadline constraint. We consider the 2 cases separately and show that in either case there will be a cycle of length $<\epsilon$ in the constraint graph.

1. Delay constraint of $P(0<P)$ between $e_{i}$ and $e_{j}$. Since this has been violated, the separation between $e_{i}$ and $e_{j}$ is less than $P$, i.e.,

$$
@\left(e_{j}\right)-@\left(e_{i}\right)<P
$$

Since the local clock of $e_{j}$ can be ahead of the local clock of $e_{i}$ by a maximum of $\epsilon$, the constraint can be violated if

$$
@\left(e_{j}\right)-@\left(e_{i}\right)<P+\epsilon
$$

or,

$$
@\left(e_{j}\right)<@\left(e_{i}\right)+P+\epsilon
$$

This implies that there is a path from $e_{i}$ to $e_{j}$ of length $Q$, where $Q<P+\epsilon$. Thus there is a cycle of length

$$
=Q-P<P+\epsilon-P<\epsilon
$$

2. Deadline constraint of $P(0<P)$ between $e_{i}$ and $e_{j}$. Since this has been violated, the distance between $e_{i}$ and $e_{j}$ is greater than P, i.e.,

$$
@\left(e_{j}\right)>@\left(e_{i}\right)+P
$$


Since the local clock of $e_{j}$ can be behind the local clock of $e_{j}$ by a maximum of $\epsilon$, the constraint can be violated if

$$
@\left(e_{j}\right)>@\left(e_{i}\right)+P-\epsilon
$$

This constraint implies that there is a path from $e_{j}$ to $e_{i}$ of length $-Q$, where $Q>P-\epsilon$. Thus there is a cycle of length

$$
=P-Q<P-(P-\epsilon)<\epsilon
$$

Theorem 3 implies that if processor clocks are synchronized to within $\epsilon$, we only need to find cycles of length less than $\epsilon$ in a constraint graph to detect a violation. The FloydWarshall all-pairs shortest-path algorithm (Tarjan 1983) can be used to find the length of the shortest cycles from all vertices in the constraint graph. The complexity of the algorithm is $O\left(n^{3}\right)$, where $n$ is the number of vertices in the graph. Since constraint graphs typically contain a relatively small number of nodes, the algorithm's complexity typically does not constitute a bottleneck.

\section{A Distributed Run-Time Monitor Prototype}

We have implemented a prototype of the distributed monitoring run-time system on a network of IBM RS/ 6000 workstations connected to a token-ring. The workstations run AIXv.3, a Unix variant that supports the assignment of static priorities to real-time processes, which can immediately preempt other user processes. Real-Time processes in AIXv.3 have higher priority than all other user processes, and the scheduler allows a higher priority real-time process to preempt a lower priority real-time process immediately. Furthermore, a fine-precision timer facility is supported, and the hardware clock registers in the RS/6000 can be read directly by a user process, which makes it possible to differentiate local events that occur $1 \mu s$ apart.

The various components of the distributed monitor and their communication links are shown in Figure 9. The clock synchronization layer synchronizes the workstation clocks to within $4 \mathrm{~ms}$. The run-time system for the distributed monitor uses the synchronized clocks for timestamping events, and performs several functions. It keeps track of the history of known events, transmits events of interest to other processors, and checks for constraint violations. The application-level processes run on top of this run-time system and are notified when a constraint violation occurs. All communication between components on the same processor, such as event history access and the reading of the synchronized clock, is via shared memory. Message-passing is used only for inter-processor communication. The monitor system, the clock synchronization layer and an $\mathrm{X}$ window system based userinterface consist of around 8000 lines of $\mathrm{C}$ code. We next describe in detail the principal layers that comprise our testbed.

We implemented the probabilistic clock synchronization algorithm described in (Cristian 1989). Every processor runs a clock-slave process that synchronizes its local clock 


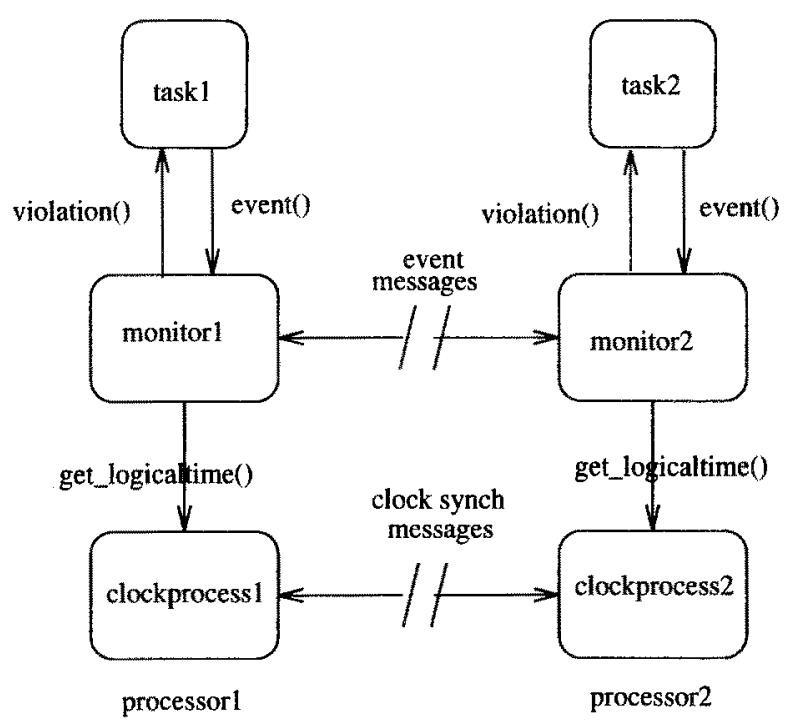

Figure 9. The Layered Interfaces of the Distributed Run-time Monitor

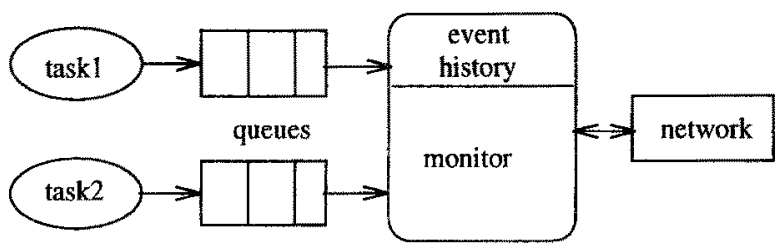

Figure 10. Application Process Interface to its Local Monitor

with the clock on a master processor by periodically exchanging messages with the clockmaster process. Based on our conservative choice of clock synchronization parameters, we calculated the clock deviation of three RS/6000's on a single token ring. The maximum master-to-slave deviation for three RS/6000's on the same token-ring is $2 \mathrm{~ms}$. The maximum slave to slave deviation was twice the master to slave deviation, i.e., $4 \mathrm{~ms}$.

The run-time system for the distributed monitor consists of a set of cooperating monitor processes, one on each processor. Each monitor process maintains the set (or subset) of constraints being monitored, registers events of local application tasks, checks for constraint violations and transmits events of interest to other monitors (including violations).

Application tasks inform the local monitor of an event occurrence by putting the event into a queue in shared memory (Figure 10). A separate queue is maintained for each application task. The monitor keeps the events in an event history that is local to the monitor. 
If the occurrence time of an event has to be sent to a remote monitor, the monitor puts the event and its local occurrence time into a message and sends it to other monitor processes. Similarly, the monitor receives events from other monitors. If a message arrives from a remote monitor or a timeout occurs, a monitor runs the satisfiability checker using Theorem 3. If a violation is detected, it notifies the application task (with termination as the default action). If there is no violation, a timeout is set based on Lemma 1. The monitor process on each processor executes the following loop:

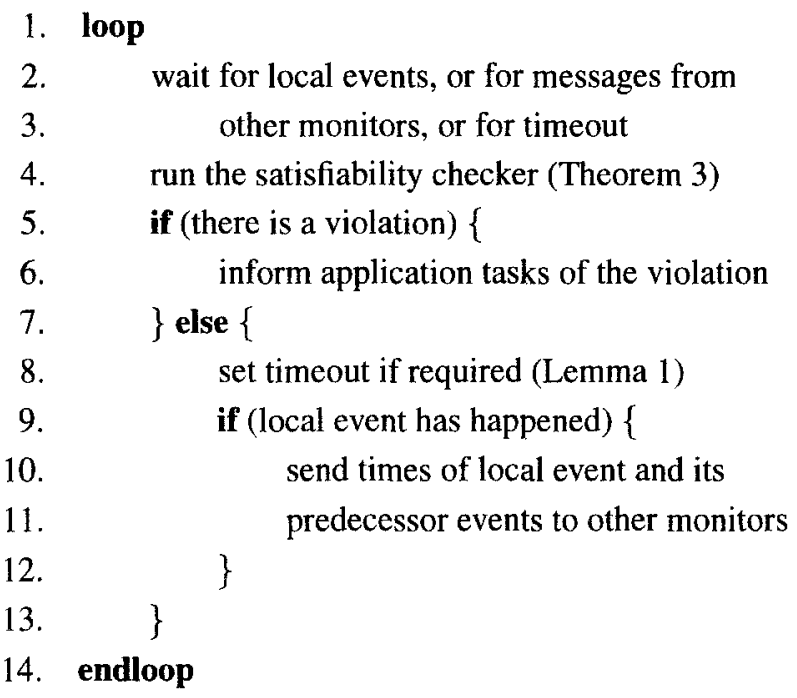

\subsubsection{Granularity of Timestamps}

Each occurrence of an event needs to be timestamped and requires the reading of the local clock. There are 2 ways of reading the hardware clock on the RS/6000 workstations. The first is the traditional means of using the POSIX interface to read the clock. The RS/6000 workstations also support an efficient assembly-language interface to read the hardware clock registers using a small number of machine instructions. Reading the clock registers directly is not a general solution because it may not be supported on other machines. On a Model 930, the POSIX call takes $23 \mu$ s while the assembly language interface takes only $512 \mathrm{~ns}$. On a Model 530, the corresponding numbers are $46 \mu s$ and $768 n s$. These results indicate that it is possible to differentiate local events that are less than $1 \mu s$ apart by directly reading the hardware registers.

\section{Conclusions}

Run-time monitoring of a distributed real-time system must address issues such as constraint specification, clock synchronization, timer granularity, message overhead and time of detection. In this paper we have extended the uniprocessor monitoring model of 
(Chodrow, Jahanian and Donner 1991) to a distributed real-time system. The principal advantage of our approach is that derived intermediate constraints can predict the violation of a user-level constraint even before the violation occurs. This can enable the application to take corrective action to adapt to the error condition. We have shown that the problem of minimizing of the number of messages exchanged between processors while still detecting violations at the earliest possible time is intractable. However, for one common class of constraints which arises whenever processing occurs in pipelined stages, message-passing requirements can be readily minimized. The drift among the various processor clocks can also be taken into account with clock synchronization. We have proved that by sending messages only along the precedence edges, all timing violations will be detected at the earliest possible time. Finally we have described a prototype implementation of our distributed monitor model.

Several extensions of this work are currently under investigation. Predictability is an important requirement of real-time systems. Therefore, one must quantify the intrusiveness of the monitoring activities on the timing behavior of the real-time application. Monitoring activities must themselves be scheduled and included in a scheduling analysis of the system (Jahanian and Rajkumar 1991). A clean high-level programming interface is needed to specify the monitored constraints and their. communication requirements. We are also designing a suite of complementary tools that support specification, testing, fault-injection and run-time monitoring of embedded real-time systems.

The approach to run-time monitoring of real-time systems, presented in this paper, has been used to check the simulation of an executable specification. References (Raju 1994), (Raju and Shaw 1994) describe the design, implementation and some experiments with a monitor for a real-time executable specification language called Communicating RealTime State Machines. The simulation of an executable specification generates a trace of events. The trace can be tested for functional and liming correctness. The advantage of monitoring a simulation is that the specification designer need not manually observe the simulation to find errors. The monitor reports errors as soon as they occur and off-line analysis is not required. Thus the specification can be debugged before moving to design and implementation. Assuming that a specification has been implemented in software, the question arises: how does one check the consistency between the specification and its implementation? One approach is to execute the specification and implementation using the same test cases and to compare the results. Our monitoring methodology permits an alternate and perhaps simpler approach: monitor the same assertions in both the specification and implementation.

\section{Acknowledgements}

We would like to thank Alan Shaw for his comments on an earlier draft of this paper. We would also like to thank the reviewers of a shorter version of this paper that appeared in the 13th RTSS. 


\section{Appendix}

Proof of Lemma 1: We first prove a lemma regarding violations in a constraint graph when all the events occur on a single processor.

LEMMA 3 A negative cycle in a constraint graph implies a constraint violation and vice versa.

Proof of Lemma 3: In the following, @(e) refers to the occurrence time of event $e$.

If part: Let there be a negative cycle of length $l$ in the constraint graph. Consider a node $e_{j}$ in the cycle. The negative cycle implies

$$
@\left(e_{j}\right)+l<@\left(e_{j}\right)
$$

This can never be satisfied. Hence, a violation has occurred.

Only if part: Say a constraint violation has occurred. The constraint can either be a deadline or delay violation. In either case we will show the existence of a negative cycle.

Delay: Consider a delay $P$ between $e_{i}$ and $e_{j}$. In order for the delay to be violated, $e_{j}$ must occur less than $P$ units after $e_{i}$, i.e.

$$
@\left(e_{j}\right)-@\left(e_{i}\right)<P
$$

Hence, there is an edge from $e_{i}$ to $e_{j}$ of length $Q$ where $Q<P$. Thus there is a cycle of length

$$
=Q-P<0
$$

Deadline: Consider a deadline $P$ between $e_{i}$ and $e_{j}$. In order for the deadline to be violated $e_{j}$ must occur later than $P$ units after $e_{i}$, i.e.

$$
@\left(e_{j}\right)>@\left(e_{i}\right)+P
$$

Hence, there is an edge from $e_{j}$ to $e_{i}$ of length $-Q$ where $Q>P$. Thus there is a cycle of length

$$
=P-Q<0
$$

\section{Proof of Lemma 1:}

Part 1. First we show that if $e_{n}$ happens before time $T$ then the constraint graph is violated. Next, we show that there cannot exist a time $Q$, where $Q<T$, such that the constraint is violated and event $e_{n}$ has not happened yet.

Say $e_{n}$ happens at $t<T$. This implies that we can insert an edge of weight $t$ from vertex 0 to $e_{n}$. The relevant portion of the constraint graph is shown in Figure AA.1. There is a cycle of length $t-T$ which is negative. Hence, the constraint graph is violated. 


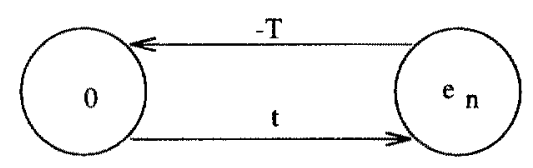

Figure A.1.

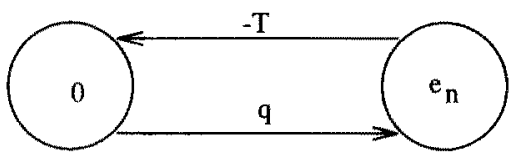

Figure A.2.

Now assume that $e_{n}$ has not happened, but there is a violation at time $Q$ before $T$. By Lemma 2 , there must exist a negative cycle at time $Q$. Let the length of path from vertex 0 to vertex $e_{n}$ be $q$ (Figure AA.2). Now,

$$
\begin{aligned}
& -T+q<0 \\
& \Rightarrow q<T
\end{aligned}
$$

This means that vertex $e_{n}$ has happened at a time less than $T$. This contradicts the assumption that $e_{n}$ has not happened before $T$. Hence, no such $Q$ can exist.

Part 2. Let the path length $P$ from vertex 0 to vertex $e_{n}$ be the minimum of all shortest paths from vertex 0 to all other vertices. First we prove that if $e_{n}$ does not happen by time $P$, then a constraint has been violated. Next, we show that there cannot exist a time $Q$, where $Q<P$, such that if any event $e_{k}$ does not occur by $Q$ then the constraint graph is violated.

If $e_{n}$ does not happen at time $P$, then $e_{n}$ can happen at $P+\alpha$ or later ( $\alpha$ is infinitesimally small). Hence we can insert an edge of weight $-(P+\alpha)$ from vertex $e_{n}$ to vertex 0 (Figure AA.3). There is a cycle from vertex 0 to vertex $e_{n}$ and back to vertex 0 of length,

$$
P-P-\alpha=-\alpha
$$

Hence the constraint graph is violated.

Now assume that there is a time $Q$, such that if some event $e_{k}$ does not happen by $Q$, then the constraint graph is violated. At time $Q$, there exists a negative cycle involving $e_{k}$, since the constraint has been violated. Since $e_{k}$ has not happened by $Q$, an edge of weight $-(Q)$ 


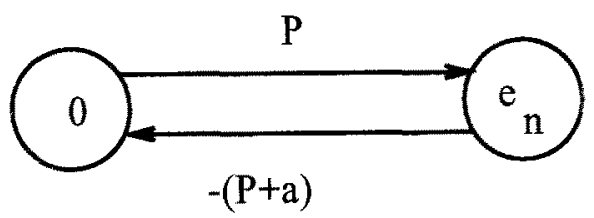

Figure A.3.

from $e_{k}$ to vertex 0 can be added. Say the path from vertex 0 to vertex $e_{k}$ is $P^{\prime}$. Since there is a negative cycle,

$$
\begin{aligned}
& P^{\prime}-Q<0 \\
& \Rightarrow P^{\prime}<Q
\end{aligned}
$$

Since $Q<P$,

$$
\Rightarrow P^{\prime}<P
$$

Hence there is path from vertex 0 to vertex $e_{k}$ that is shorter than $P$. Thus $P$ is not the minimum of shortest paths from vertex 0 to all other vertices. This is a contradiction. Hence no such $\mathrm{Q}$ can exist.

\section{Notes}

1. RS/6000 and AIX are trademarks of IBM Corporation.

2. In a real system, the signal may be sent to one of many consoles. In this paper, we assume a single console for the sake of simplicity.

3. We assume that indices are not arithmetical expressions.

\section{References}

Arvind, K. 1989. A New Probabilistic Algorithm for Clock Synchronization, Proc. IEEE Real-Time Systems Symp., Santa Monica, pp. 330-339.

Babaoglu, O. and Marzullo, K. 1993. Consistent Global States of Distributed Systems: Fundamental Concepts and Mechanism. in Distributed Systems, S. Mullender (editor), 2nd edition.

Chandy, K. M. and Lamport, L. 1985. Distributed Snapshots: determining global states of distributed systems. ACM Transactions on Computer Systems.

Chodrow, S., Jahanian, F. and Donner, M. 1991. Run-Time Monitoring of Real-Time Systems, Proc. IEEE Real-Time Systems Symp., pp. 74-83.

Cristian, F. 1989. Probabilistic Clock Synchronization, Distributed Computing. 3: 146-158.

Garey, M. R. and Johnson, D. S. 1979. Computers and Intractability: A Guide to the Theory of NP.Completeness, W. H. Freeman and Company. 
Garg, V. and Waldecker, B. 1992. Unstable Predicate Detection in Distributed Programs, Technical Report, University of Texas at Austin.

Haban, D. and Shin, K. G. Dec. 1989. Application of Real-Time Monitoring to Scheduling Tasks with Random Execution Times, Proc. IEEE Real-Time Systems Symp., pp. 172-181.

Haban, D. and Wybranietz, D. Feb. 1990. A Hybrid Monitor for Behavior and Performance Analysis of Distributed Systems. IEEE Trans. on Software Eng. (16)2: 197-211.

Jahanian, F. and Goyal, A. June 1990. A Formalism for Monitoring Real-Time Constraints at Run-time, Proc. IEEE Fault-Tolerant Computing Symp., pp. 148-155.

Jahanian, F. and Rajkumar, R. May 1991. An Integrated Approach to Monitoring and Scheduling in Real-Time Systems, IEEE Workshop on Real-Time Operating Systems and Sofware.

Kenny, K. B. and Lin, K.-I. May 1991. Building Flexible Real-Time Systems using the Flex Language. Computer, pp. $70-78$.

Kilpatrick, C., Schwan, K. and Ogle, D. March 1990. Using Languages for Capture, Analysis and Display of Performance Information for Parallel and Distributed Applications, International Conf. on Computer Languages.

Koo, R. and Toueg, S. January 1987. Checkpointing and Rollback-recovery for Distributed Systems. IEEE Transactions on Software Engineering, pp. 23-31.

Kopetz, H. and Verissimo, P. 1993. Real-Time and Dependability Concepts, Distributed Systems, S. Mullender(editor), 2nd Edition, pp. $411-46$.

Lundelius, J. and Lynch, N. 1984. An Upper and Lower Bound for Clock Synchronization, Information and Control 62, pp. 190-204.

Marzullo, K. and Neiger, G. 1991. Detection of Global State predicates, Proc. of 5 th International Workshop on Distributed Algorithms (WDAG-91), Delphi, Greece, Springer-Verlag.

Marzullo, K. and Sabel, L. March 1992. Using Consistent Subcuts for Detecting Stable Properties, Technical Report Department of Computer Science, Cornell University.

Raju, S. C. V. 1994. Using Assertions for Validating, Verifying and Monitoring Real-Time Systems, Ph.D. Thesis, University of Washington.

Raju, S. C. V., Rajkumar, R., and Jahanian, F. December 1992. Monitoring Timing Constraints in Distributed Real-Time Systems, Proceedings of the 14th Real-Time Systems Symposium, pp. 57-67.

Raju, S. C. V. and Shaw, A. C. February 1994, "A Prototyping Environment for Specifying, Executing and Checking Communicating Real-Time State Machines", Software - Practice \& Experience, pp. 175-195.

Snodgrass, R. 1988. A Relational Approach to Monitoring Complex Systems. ACM Trans. on Computer Systems. (6) $2: 157-196$

Tarjan, R. E. 1983. Data Structures and Network Algorithms, Society for Industrial and Applied Mathematics.

Tokuda, H., Koreta M. and Mercer, C. W. Jan. 1989. A Real-Time Monitor for a Distributed Real-Time Operating System, ACM Sigplan Notices. (24)1: 68-77.

Tsai, J. P., Fang, K-Y and Chen, H-Y. 1990. A Noninvasive Architecture to Monitor Real-Time Distributed Systems, IEEE Computer. (23)3: 11-23. 